\title{
rBMSCs/ITGA5B1 Promotes Human Vascular Smooth Muscle Cell Differentiation via Enhancing Nitric Oxide Production
}

\author{
Yingxin Zhang ${ }^{1}$, Jie Ding ${ }^{1}$, Cong Xu${ }^{1}$, Hongli Yang ${ }^{1}$, Peng Xia ${ }^{2}$, Shengjun $\mathrm{Ma}^{2}$, Haiying Chen $^{1}$ \\ ${ }^{I}$ Central Laboratory of Liaocheng People's Hospital, Liaocheng, Shandong, China, \\ ${ }^{2}$ Department of Cardiology, Liaocheng People's Hospital, Liaocheng, Shandong, China
}

Background and Objectives: Previous studies have shown that integrins alpha5betal (ITGA5B1) gene-modified rat bone marrow mesenchymal stem cells (rBMSCs) could prevent cell anoikis and increase the nitric oxide (NO) production. Here we examined the capability of rBMSCs/ITGA5B1 on the phenotype modulation of Human Pulmonary Artery Smooth Muscle Cell (HPASMC) in vitro.

Methods and Results: The synthetic (dedifferentiated) phenotype of HPASMC was induced by monocrotaline (MCT, $1 \mu \mathrm{M}$ ) for $24 \mathrm{~h}$ and then co-cultured with rBMSCs/TTGA5B1 in a transwell culture system. The activation of NO/cGMP (nitric oxide/Guanosine-3', 5'-cyclic monophosphate) signaling was investigated in HPASMC. The changes of pro-inflammatory factors, oxidative stress, vasodilator, vasoconstrictor, contractile and synthetic genes, and the morphological changes of HPASMC were investigated. The results of this study showed that the NO/cGMP signal, endothelial nitric oxide synthase (eNOS) expression, the expression of the vasoprotective genes heme oxygenase-1 (HMOXl) and prostaglandin-endoperoxide synthase 2 (PTGS2) were increased, but the expression of transforming growth factor- $\beta 1$ (TGF$\beta$ 1), CCAAT/enhancer-binding proteins delta (Cebpd), Krüppel-like factor 4 (KLF4), and activating transcription factor 4 (ATF4) were reduced in MCT treated HPASMC co-cultured with rBMSCs/ITGA5B1. The synthetic smooth muscle cells (SMCs) phenotype markers thrombospondin-1, epiregulin and the vasoconstrictor endothelin (ET)-1, thromboxane A2 receptor (TbxA2R) were down-regulated, whereas the contractile SMCs phenotype marker transgelin expression was up-regulated by rBMSCs/ITGA5B1. Furthermore, rBMSCs/ITGA5B1 promoted the morphological restoration from synthetic (dedifferentiation) to contractile (differentiation) phenotype in MCT treated HPASMC.

Conclusions: $\mathrm{rBMSCs} / \mathrm{ITGA5B1}$ could inhibit inflammation and oxidative stress related genes to promote the HPASMC cell differentiation by activation $\mathrm{NO} / \mathrm{cGMP}$ signal.

Keywords: Bone marrow derived mesenchymal stem cells, Integrin, Nitric oxide, Phenotypic transition, Human vascular smooth muscle cell (HPASMC)

\footnotetext{
Received: September 4, 2018, Revised: September 6, 2018, Accepted: October 8, 2018, Published online: November 30, 2018 Correspondence to Haiying Chen

Central Laboratory of Liaocheng People's Hospital, Liaocheng, Shandong 252000, China

Tel: +86-134-6576-9078, Fax: +86-635-827-6009

E-mail: hychenmay5@126.com

Co-Correspondence to Shengiun $\mathrm{Ma}$

Department of Cardiology, Liaocheng People's Hospital, Liaocheng, Shandong 252000, China

Tel: +86-134-6576-9078, Fax: +86-635-827-2289

E-mail: msj6336@126.com.

(a) This is an open-access article distributed under the terms of the Creative Commons Attribution Non-Commercial License (http://creativecommons.org/licenses/by-nc/4.0/), which permits unrestricted non-commercial use, distribution, and reproduction in any medium, provided the original work is properly cited.

Copyright (c) 2018 by the Korean Society for Stem Cell Research
} 


\section{Introduction}

Pulmonary arterial hypertension $(\mathrm{PAH})$ is a vascular remodeling disease with a poor prognosis and limited therapeutic option . Pulmonary vascular remodeling is the key structural alteration in the normal architecture of the walls of pulmonary arteries. Vascular smooth muscle cells (SMCs) are involved in this process (1).

Vascular SMCs exhibit phenotypic and functional plasticity in response to environment. Upon vascular injury, SMCs undergo phenotypic transition from a quiescent contractile (differentiated) to a proliferative/migratory synthetic (dedifferentiated) phenotype. This processes plays a major role in the development of vascular remodeling (2), and is accompanied by decrease in expression of SMCs-specific markers (transgelin, SM a-actin) responsible for SMCs contraction, increase in expression of synthetic SMC markers (thrombospondin, epiregulin), and production of pro-inflammatory mediators that modulate induction of proliferation and chemotaxis (3). Inflammatory infiltrates into the lungs mediate phenotypic transition in pulmonary vascular SMCs, stimulate the structural remodeling in the vasculature (4), lead to the activation of oxidative stress, cause a decrease in vasodilators (nitric oxide, prostacyclins) and an increase in vasoconstrictors endothelin (ET)-1 and thromboxanes (4).

Some studies suggest that nitric oxide (NO) has been shown to reduce inflammation, apoptosis (5), and regulate the vascular SMCs proliferation, migration and differentiation (6). NO/cGMP (cyclic 3'-5' guanosine monophosphate) signaling plays a critical role in smooth muscle tone, cardiac contractility, and cell growth (7). However, the disturbance of the endothelial cells in PAH results in the alteration of NO/cGMP signaling, then lead to a decrease in endothelial nitric oxide synthase (eNOS) expression and NO production (8). Therefore, it is important to maintain the functions of endothelial cells.

Mesenchymal stem cells (MSCs) have been proposed to the treatment of pulmonary hypertension to improve vascular endothelial functions of the monocrotaline (MCT)injured lung. MCT is a pyrrolizidine alkaloid which selectively injures the pulmonary vascular endothelium and induces pulmonary vasculitis, can cause PAH in rats and is widely utilized to analyze the pathophysiology of PAH (9). Fischer reported that the majority of MSCs were trapped inside the lungs following intravenous delivery (10), and MSCs can suppress ongoing inflammation (11), these may benefit for the treatment of lung diseases. Our previous study had found that integrin alpha5 (ITGA5), integrin betal (ITGB1) increased the NO production and prevented the cell anoikis (12) in vitro. In present study, we focus on the effect of rBMSCs/ITGA5B1 on MCT induced phenotypic transition in co-cultured HPASMC (Pulmonary Artery Smooth Muscle Cells) to evaluate its therapeutic potential for the future treatment of PAH.

\section{Materials and Methods}

\section{Ethics statement}

The adult male Wistar rats weighing $100 \sim 120 \mathrm{~g}$ (certificate number SCXK Shandong 20140007) were obtained from the animal experimental center of Shangdong University (Shandong, China). The study was approved by the Institutional Animal Care and Use Committee (Liaocheng People's Hospital, Shandong, China). All experiment with animals were conducted in accordance with the guidelines described in the National Institutes of Health "Guide for the Care and Use of Laboratory Animals".

\section{Cells and Reagents}

HPASMC were purchased from Sciencell, cultured with smooth muscle cell medium with smooth muscle cell growth supplement (Sciencell Research Laboratories, Inc., San Diego, CA, USA). The adult male Wistar rats were used as rBMSCs donors. The femurs and tibiae were removed from the rats and rBMSCs were isolated and cultured as we described previously (13). The pLVXmCMV-mCherry lentiviral vector backbone, the ITGA5, ITGB1 gene over-expressed lentiviral vectors pLVXmCMV-ITGA5-mCherry (LV-ITGA5) and pLVX-mCMVITGB1-mCherry (LV-ITGB1) were purchased from Biowit Technologies Co., Ltd. (Shenzhen, China). The lentiviral packaging plasmids VSV-G, psPax2 and pRSV-Rev were given as a kind gift by Dr Padraig Strappe (Central Queensland University, Australian).

\section{Transduction, co-culture and the morphological changes of HPASMC}

The Lentiviral vectors production and transduction was performed as we described previously (13). rBMSCs were randomly divided into two groups for co-culture: vector group (infected with empty vector virus), and rBMSCs/ ITGA5B1 group (co-infected with ITGA5 and ITGB1 virus). The transwell co-culture system was used for HPASMC and rBMSCs co-culture, briefly, HPASMC was cultured in the lower compartment of a transwell-plate (Costar 3412, Corning Incorporated, NY, USA), treated with MCT $(1 \mu \mathrm{M})$ for $24 \mathrm{~h}$ after HPASMC reached 50 $60 \%$ confluence, and named for MCT-HPASMC. Each group of rBMSCs (5 days post transduction) was placed 
onto $0.4 \mu \mathrm{m}$ pore size polycarbonate membranes of the upper compartment in the transwell plate. HPASMC were randomly divided into four groups: Control group (only HPASMC), Model group (only MCT-HPASMC), Vector group (MCT-HPASMC in lower compartment and co-cultured with rBMSCs/Vector), ITGA5B1 group (MCTHPASMC in lower compartment and co-cultured with rBMSCs/ITGA5B1). The morphological changes of each group of HPASMC were investigated and photographed under light microscopy (CKX71, Olympus) after co-cultured for $72 \mathrm{~h}$.

\section{NO detection}

Nitrite (the stable end product of NO) was measured by a Nitric Oxide Colorimetric Assay Kit (Biovision, Milpitas, CA, USA) in each group of HPASMC supernatants using the Griess reaction as described in the previous studies (14). The absorbance was measured by microplate reader (Multiskan MK3, Thermo scientific, USA) at $540 \mathrm{~nm}$. The concentration of nitrite in HPASMC supernatants was calculated by a calibration curve prepared from serial dilutions of nitrite standard solution. Each sample was analyzed in triplicate and each experiment was repeated for three times.

\section{Measurement of reactive oxygen species (ROS) levels}

Reactive Oxygen Species Assay Kit (Beyotime, Jiangsu, China) was used for detecting the intracellular ROS levels by the oxidation-sensitive fluorescent probe 2', 7'-dichlorofluorescein diacetate (DCFH-DA). Cells were washed twice with PBS and incubated with DCFH-DA (10 $\mu \mathrm{M})$ for $20 \mathrm{~min}$ after HPASMC co-cultured with rBMSCs/ ITGA5B1for $72 \mathrm{~h}$. ROS can oxidize non fluorescent DCFH to produce fluorescent DCF. The cellular production of fluorescent DCF was measured with flow cytometry (Becton, Dickinson) and analyzed by the BD FACSDiva software.

\section{Western blotting}

To determine the protein expression of eNOS, CCAAT/ enhancer-binding proteins delta (Cebpd), Krüppel-like factor 4 (KLF4), transforming growth factor- $\beta 1$ (TGF- $\beta$ 1 ), and endothelin (ET)-1, total protein was extracted in ice-cold lysis buffer containing PMSF (Beyotime, Jiangsu, China) for $30 \mathrm{~min}$ on ice at 5 days post-transduction. The lysate was then centrifuged at $12,000 \times \mathrm{g}$ for $5 \mathrm{~min}$ at $4^{\circ} \mathrm{C}$. The supernatant was collected and the protein concentration was determined by BCA Protein Assay Kit (Beyotime, Jiangsu, China). Equal amounts of protein (20 $\mu \mathrm{g})$ were separated by $10 \%$ SDS-PAGE, and then were electro-transferred to polyvinylidene difluoride (PVDF) membranes (Millipore, Bedford, MA). The membranes were blocked with 5\% skimmed milk at room temperature for $1 \mathrm{~h}$, and then incubated with the primary monoclonal anti-eNOS (BD Biosciences PharMingen, San Diego, CA, USA), anti-Cebpd, anti-TGF- $\beta 1$, anti-ET-1, polyclonal anti-KLF4 antibody (1:1000, all from Abcam, Cambridge, $\mathrm{UK}$, and monoclonal anti- $\beta$-actin antibody (1:1000, Beyotime, Jiangsu, China) respectively overnight at $4^{\circ} \mathrm{C}$. The membranes were washed three times for $5 \mathrm{~min}$ in TBST and then incubated with goat anti-rabbit or goat anti-mouse IgG/HRP conjugated secondary antibodies (1:1000) for $1 \mathrm{~h}$. The protein bands were visualized using ECL kit (Beyotime, Jiangsu, China). Images were obtained and analyzed with AlphaView analysis system (ProteinSimle, USA). The expression of $\beta$-actin was used as an internal control.

\section{Quantitative PCR (qPCR)}

Total RNA in HPASMC was extracted by Trizol reagent (Thermo Fisher Scientific), then RNA (1 $\mu \mathrm{g})$ was reverse-transcribed using a Prime Script ${ }^{\mathrm{TM}}$ RT reagent Kit with gDNA Eraser in $20 \mu 1$ reactions. The cDNA product was diluted 3-folds, aliquoted, and stored at $-80^{\circ} \mathrm{C}$. Amplification was performed on ABI 7500 Real-Time PCR system (Applied Biosystems) using SYBR ${ }^{\circledR}$ Premix Ex Taq $^{\mathrm{TM}}$ II kit (all from Takara, Dalian, China). The thermocycling conditions were $30 \mathrm{~s}$ pre-incubations at $95^{\circ} \mathrm{C}$ for one cycle, followed by 40 cycles of $95^{\circ} \mathrm{C}$ for $5 \mathrm{~s}$ and $60^{\circ} \mathrm{C}$ for $34 \mathrm{~s}$. Primers for guanosine-3',5'-cyclic monophosphate (cGMP) were 5'-TGG AGG AGA ATA CTG GCA AGG-3' (Sense) and 5'-TGG CTC TCT CCA CTG CTT CA-3' (antisense), activating transcription factor 4 (ATF4), 5'-CCA ACA ACA GCA AGG AGG AT-3' (Sense), 5'-AGG TCA TCT GGC ATG GTT TC-3' (antiSense), heme oxygenase-1 (HMOX1), 5'-AAC TTT CAG AAG GGC CAG GT-3' (Sense), 5'-GAA GAC TGG GCT CTC CTT GTT-3' (antiSense), prostaglandin-endoperoxide synthase 2 (PTGS2), 5'-GTC TGG TGC CTG GTC TGA TG-3' (Sense), 5'-GCC TGC TTG TCT GGA ACA AC-3' (antiSense), thromboxane A2 receptor (TbxA2R), 5'-TCG CTA CAC CGT GCA ATA CC-3' (Sense), 5'-TGT TCA GCA GGA AGG ACA GC-3' (antiSense), Thrombospondin, 5'-TCC TCC TCA CCC TTG ACA AC-3' (Sense), 5'-TGG ACA GCT CAT CAC AGG AG-3' (antiSense), transgelin (TAGLN), 5'-TCC AGG TCT GGC TGA AGA AT-3' (Sense), 5'-GCT CCA TCT GCT TGA AGA CC-3' (antiSense), epiregulin, 5'-TGG TGT CCG ATG TGA ACA CT-3' (Sense), 5'-TGG AAC CGA CGA CTG TGA TA-3' (antiSense), and GAPDH, 5'-TGC ACC 
ACC AAC TGC TTA GC-3'(sense), 5'-GGC ATG GAC TGT GGT CAT GAG-3' (antisense). NO-reverse transcription controls (No-RT) and No-template controls (NTC) were performed for each RNA type and primer pair. The fold changes amplification for targeted genes was normalized to the housekeeping gene GAPDH by the $2^{-\triangle \triangle \mathrm{CT}}$ method. Samples were analyzed in triplicate and each experiment was repeated for at least three times.

\section{Statistical analysis}

Values were expressed as means \pm SD. The data were analyzed by one-way ANOVA, followed by SNK-q test. The level of statistical significance was set at $\mathrm{p}<0.05$.

A

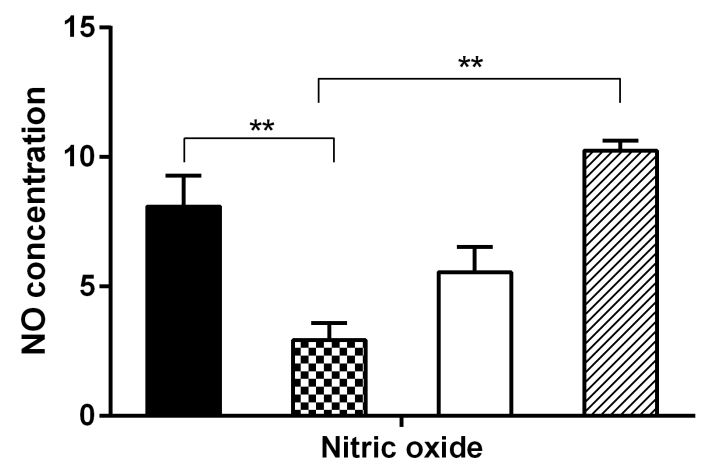

C

ROS production

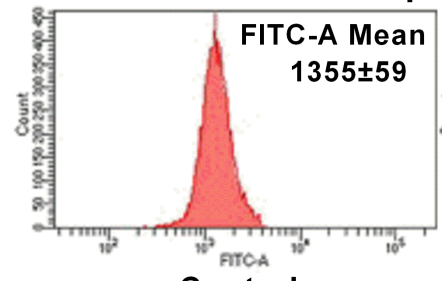

Control
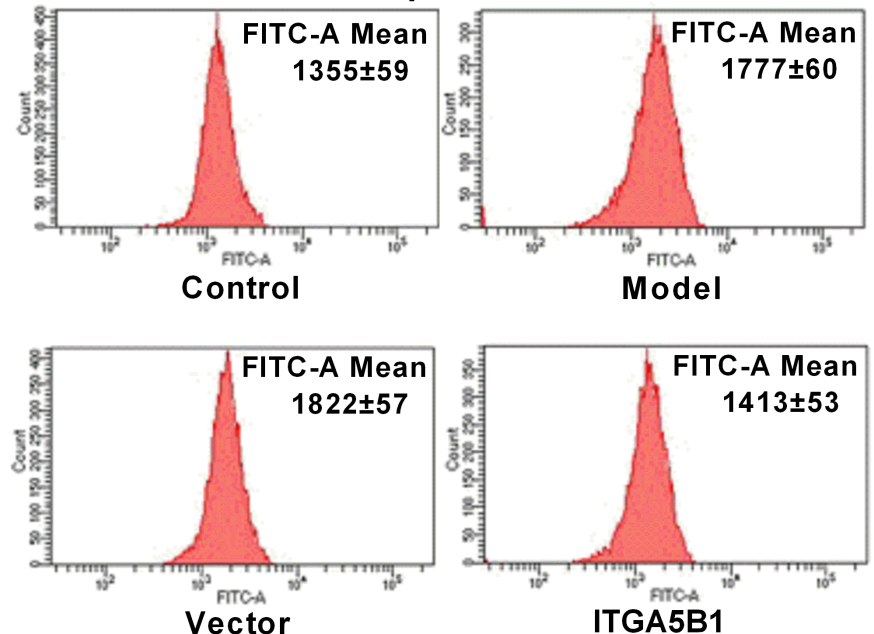

Model

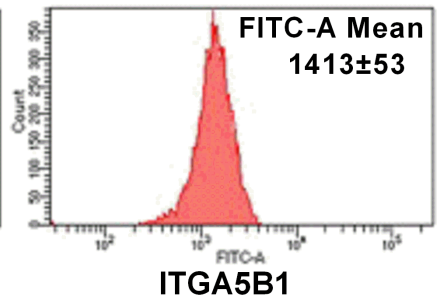

\section{Results}

rBMSCs/ITGA5Bl enchanced the NO/cGMP signal, eNOS expression and inhibited ROS production

Compared to control groups, NO concentration and cGMP mRNA expression was reduced but ROS was increased in MCT treated HPASMC model groups. However, the NO level, cGMP mRNA expression was elevated about 3.5-folds and 3-folds respectively but ROS production was reduced in HPASMC co-cultured with rBMSCs/ITGA5B1 compared to model groups (Fig. 1A C). Furthermore, eNOS, which is associated with diastolic factors NO production, was activated in ITGA5B1 groups compared to model groups (Fig. 1D).

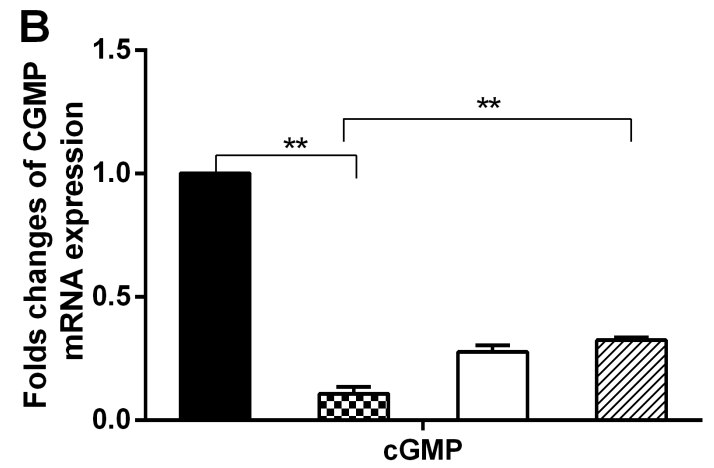

D
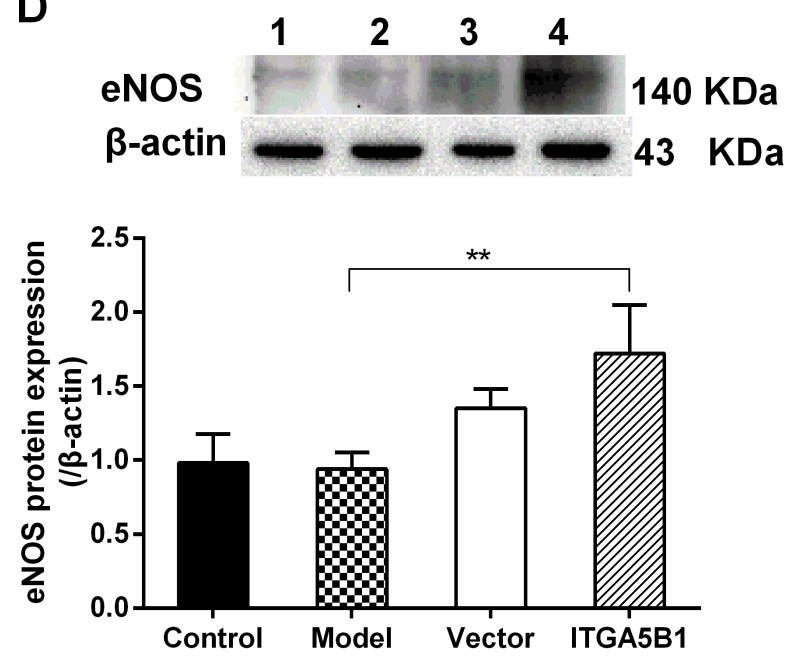

Fig. 1. The effect of rBMSCs/ITGA5B1 on NO/cGMP signal, eNOS expression and ROS production. (A) NO production was evaluated by measuring nitrite (the stable end product of $\mathrm{NO}$ ) concentration by a nitric oxide colorimetric assay. (B) The mRNA expression of cGMP. (C) ROS production. ROS level was detected by the fluorescent probe DCFH-DA. The FITC-A Mean represents for the average fluorescence intensity. (D) The expression of eNOS protein was investigated by western-blot. 1: Control groups, 2: Model groups, 3: Vector groups, 4: ITGA5B1 groups. ${ }^{*} \mathrm{p}<0.05,{ }^{* *} \mathrm{p}<0.01$. 
rBMSCs/ITGA5B regulated stress response genes, inflammation factors and diastolic-systolic factors in MCT treated HPASMC

The present study found that MCT induced the oxidative stress and inflammation factors increasing in HPASMC, manifestied by the up-regulated stress-induced transcription factor ATF4, down-regulated vasoprotective genes HMOX1 and PTGS2 mRNA expression (Fig. 2A and $2 \mathrm{~B}$ ), increased the pro-inflammation factors Cebpd, KLF4 and TGF- $\beta 1$ protein expression (Fig. 2C and 2D). After HPASMC co-cultured with rBMSCs/ITGA5B1, the expression of ATF4, Cebpd, KLF4, and TGF- $\beta 1$ were all depressed but HMOX1 and PTGS2 was elevated in HPASMC by introduction of rBMSCs/ITGA5B1 (Fig. 2). Furthermore and importantly, rBMSCs/ITGA5B1 could improve the unbalance state of diastolic-systolic factors expression of MCT treated HAPSMC. The eNOS expression and NO production were increased in ITGA5B1 groups as we mentioned above (Fig. 1A and 1D). The Tbxa2R mRNA and vasoconstrictor ET-1 protein expression was up-regulated 1.46-folds and 2.56-folds higher respectively in model groups than in control groups, but it was
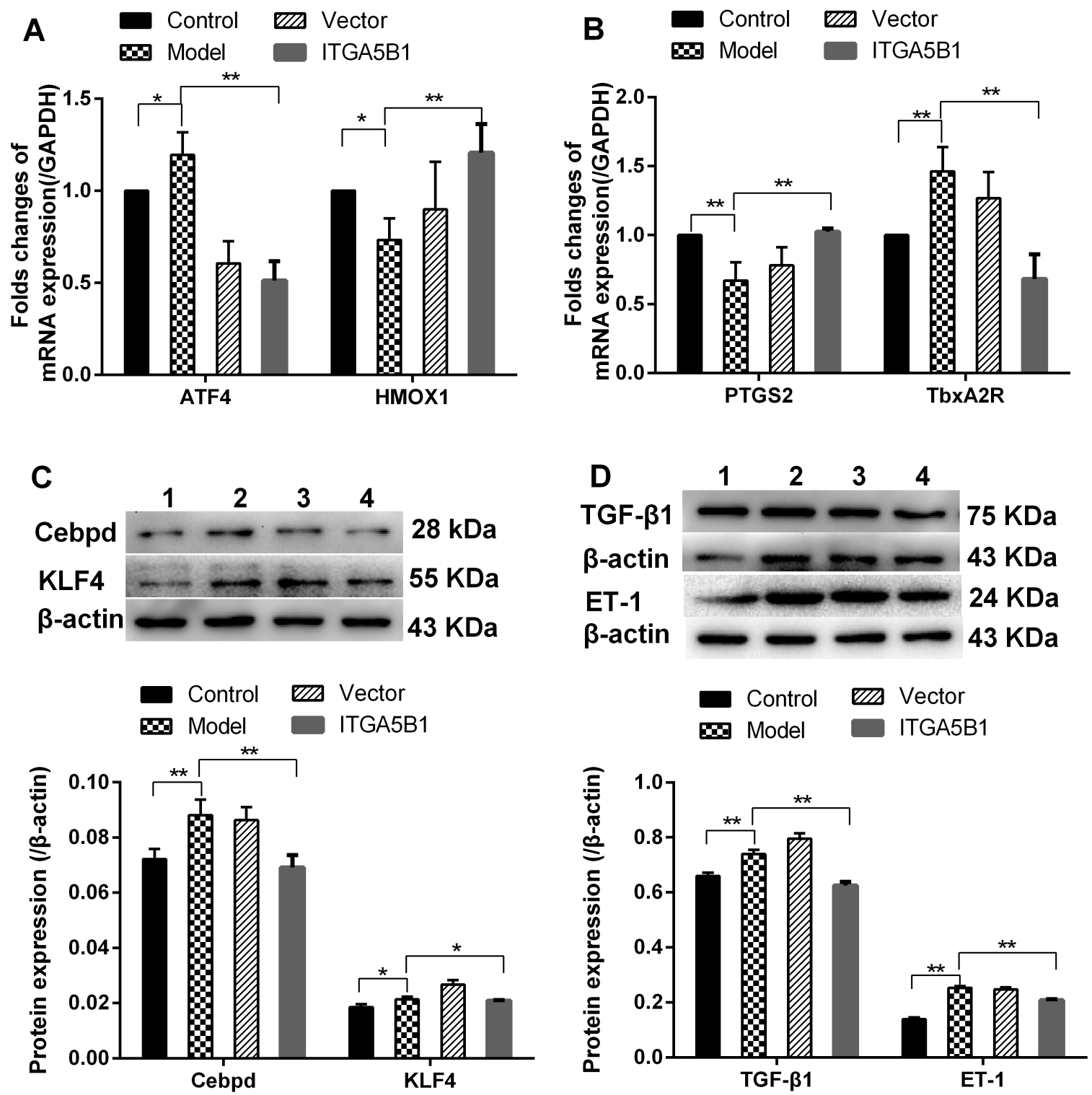

Fig. 2. The regulation effects of $\mathrm{rBMSC} / \mathrm{ITGA} 5 \mathrm{~B} 1$ on the expression of stress response genes, pro-inflammatory factors and vasoconstrictors. (A, B) The mRNA expression of ATF4, HMOX1, PTGS2 and TbxA2R. (C, D) The protein expression of pro-inflammatory factors and vasoconstrictor. 1: Control groups, 2: Vector groups, 3: Model groups, 4: ITGA5B1 groups. 
down-regulated by co-cultured with rBMSCs/ITGA5B1 (Fig. 2B and 2D).

\section{Effect of rBMSCs/ITGA5B1 on morphological changes and phenotypic transition in HAPSMC}

For normal cell morphology of HPASMC in control groups, the cells were spindle-shaped with small size, but the cell morphology and overall cell size was changed much in MCT treated HPASMC groups, which showed fibroblast like and larger in volume compared to control groups. However, rBMSCs/ITGA5B1 could rescue the MCT induced HPASMC morphologic changes, the cells morphology in ITGA5B1 groups was similar to that of the control group, showing more spindle-shaped and smaller size than in model groups (Fig. 3). Moreover, the mRNA expression of synthetic SMCs phenotype marker thrombospondin-1 and epiregulin were up-regulated significantly, increased above 1.4-folds and 100-folds respectively, whereas the transgelin was down-regulated nearly 2.5 -folds in model groups than in control groups (Fig. 4). Interestingly, MCT induced phenotypic transition from contractile to synthetic phenotype in HPASMCs was depressed by co-cultured with rBMSCs/ITGA5B1, the thrombospondin-1, epiregulin was decreased 2.88-folds and 3.17-folds respectively, but the contractile SMCs phenotype marker transgelin was increased 2.3-folds compared to model groups (Fig. 4).

\section{Discussion}

Mesenchymal stem cells (MSCs) have been proposed to the treatment of pulmonary hypertension to improve vascular endothelial functions, but the low survival rates after transplantation due to cell death via anoikis driven by the loss of cell adhesion is a major obstacle to stem cell therapy (15). Our previous studies have shown that ITGA5B1 could prevent rBMSCs cell anoikis and increase the NO production (12). In this study we demonstrated that rBMSCs/ITGA5B1 activated NO/cGMP signal, attenuated inflammation factors expression, oxidative stress, and improve the unbalance state of diastolic-systolic factors expression in MCT treated HAPSMC.

Endothelial cell dysfunction plays an important role in the pathogenesis of $\mathrm{PAH}$ and is associated with diminished eNOS-derived NO production. The endothelial barrier disruption resulted in the up-regulation of inflammatory cytokines and contribute to the development of oxidative stress, leading to generation of ROS (16). The increased ROS level can significantly inhibit the bio-availability of NO and ultimately lead to a reduction in NO-in-

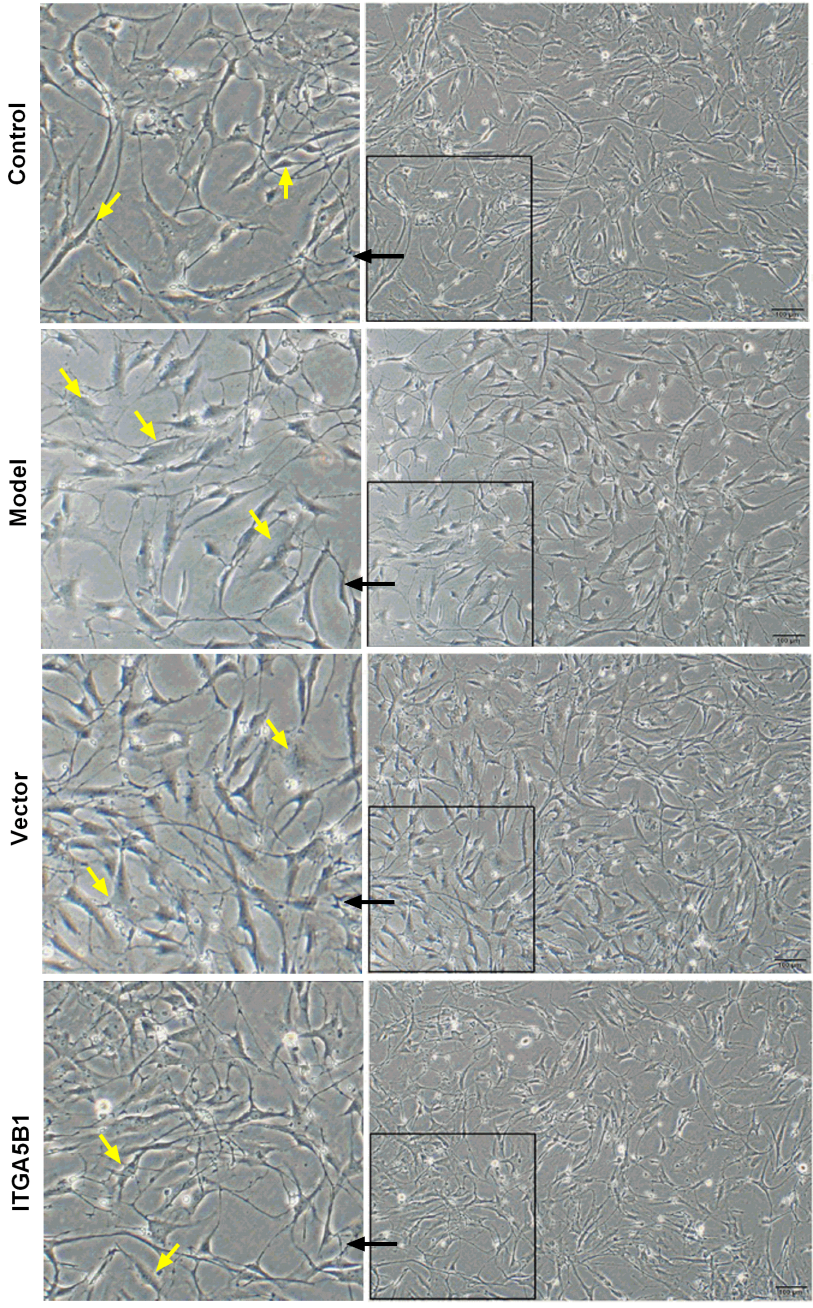

Fig. 3. Effect of rBMSCs/ITGA5B1 on morphological changes of MCT induced HPASMC. Compared to control groups, the cells showed fibroblast like and hypertrophy in model groups, whereas the cell shape in ITGA5B1 groups showed spindle-shaped with small size and similar in morphology to control groups. $\mathrm{Bar}=100 \mu \mathrm{m}$ $(10 \times)$.

duced vasodilation of the pulmonary vasculature (4). NO acts as a suppressor of SMC proliferation and endothelium derived relaxing factor, and has a great impact on the pulmonary circulation mainly through the second messenger cGMP (17). Therefore, the enhanced NO/cGMP activation by $\mathrm{rBMSCs} / \mathrm{ITGA5B1}$ may exert its regulation effect on the inflammation, oxidative stress, and diastolic-systolic factors.

The present study demonstrated that rBMSCs/ITGA5B1 depressed the expression of pro-inflammatory factors (Cebpd, KLF4 and TGF- $\beta$ 1), ROS production, stress response transcription factor ATF4, but up-regulated HMOX1 and PTGS2 expression, restore the MCT induced morphological changes and inhibited phenotypic tran- 

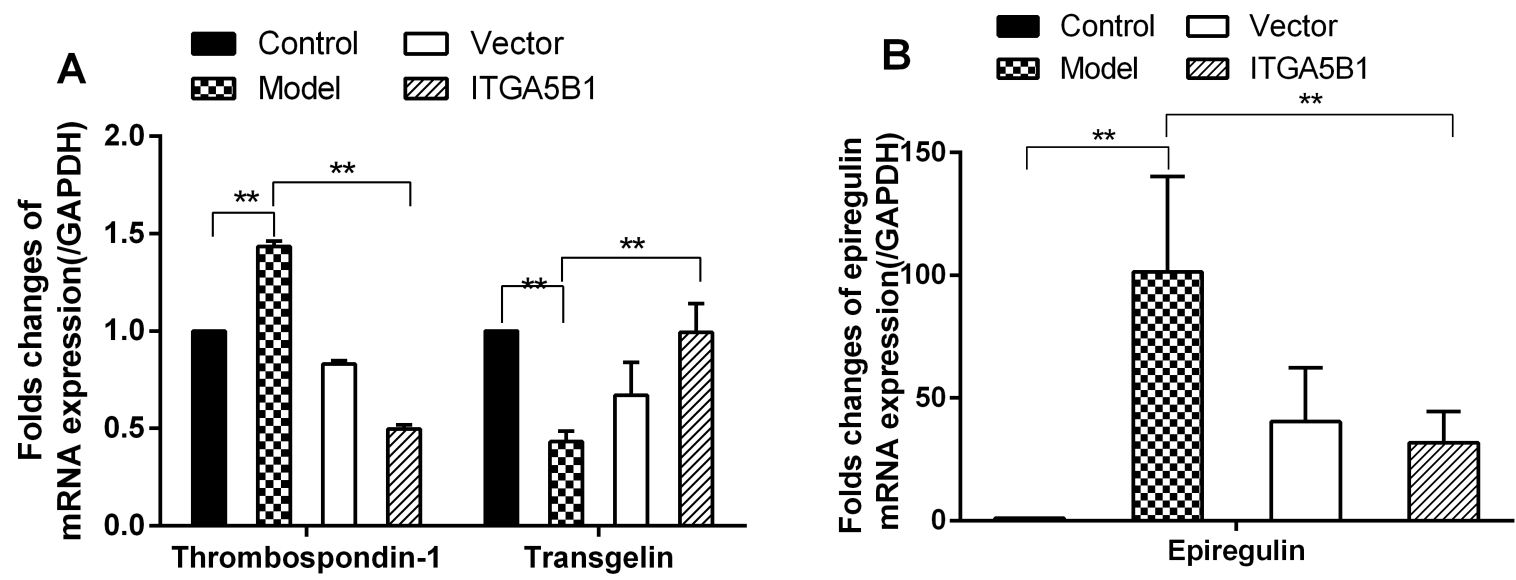

Fig. 4. The mRNA expression of the thrombospondin, transgelin and epiregulin. The synthetic SMCs phenotype markers thrombospondin and epiregulin was reduced while the contractile SMCs phenotype marker transgelin expression was up-regulated by rBMSCS/ITGA5B1.

sition in HPASMCs. It has been widely accepted inflammation contributes to pulmonary vascular remodeling, and the Cebpd, KLF4 and TGF- $\beta 1$ is capable of regulating inflammation. Cebpd belongs to the CCAAT/enhancer-binding protein family, and these proteins functions as transcription factors in many biological processes, including pro-inflammatory gene expression (18). Cebpd is a central gene in inflammation and plays an important role in the inflammatory responses (18). For transcription factor KLF4, it involved in SMC dedifferentiation. KLF4 could induce and regulate the inflammatory factors expression in macrophages (19), bound to the TGF- $\beta$ control element transgelin promoters, and suppress the SMC differentiation gene expression (20). TGF- $\beta 1$ is a multifunctional signaling protein involved in growth stimulation, migration, phenotypic transition and morphological changes of vascular SMC (21). In our study, we observed that the pro-inflammatory factors Cebpd, KLF4 and TGF- $\beta 1$ up-regulation with the increase of ROS in MCT treated HPASMC, but they were inhibited by co-cultured with rBMSCs/ITGA5B1. Furthermore, ATF4 was down-regulated and HMOXlwas up-regulated by rBMSCs/ITGA5B1. ATF4 is a master transcriptional activator of the integrated stress response, can exert its pro-inflammatory through nuclear factor-kappaB and IL-6 activation (22). HMOX1 is an oxidative stress response enzyme and generally regarded as a protective mechanism, which presents with anti-inflammatory properties and was inversely correlated to pulmonary vascular resistance (23). Recently report demonstrated that the decreased lung vasoprotective genes HMOX1 and PTGS2 associated with greater pulmonary inflammation (24). Although the overwhelming majority of studies on PTGS2 had explored the role of it as the key factor in the cellular response to inflammation, however, there was sporadic report suggested that PTGS2 was a key regulatory enzyme in the synthesis of the antifibrotic agent prostaglandin E2 (25), and exhibited vasoprotective function (24). More importantly, there is considerable data supporting the anti-inflammatory properties of MSCs will be of benefit for many conditions $(26,27)$. The concept that the pathological phenotype of SMCs from a contractile to a synthetic phenotype was triggered by different environmental stress and injury events including activated inflammatory cascades (28). Therefore, the inhibitory effect of rBMSCs/ITGA5B1 on pro-inflammation factors and oxidative stress may mitigate phenotypic transition.

In case of the vessel damage under different environmental stress, vascular SMCs are able to switch from the quiescent contractile phenotype to the pro-inflammatory synthetic phenotype. In this study, MCT induced the HPASMC with hypertrophy, enhanced synthetic SMCs phenotype markers (thrombospondin-1 and epiregulin) expression and repressed contractile SMCs phenotype marker transgelin expression in model groups compared to control groups, whereas, the morphological change and phonotypic transition of HPASMC induced by MCT was reversed by co-cultured with rBMSCs/ITGA5B1. The cell shape in ITGA5B1 groups showed similar in morphology to control groups, a single layer of cells with the elongated spindle shaped cells were clearly observed in ITGA5B1 groups, and the expression of thrombospondin-1, epiregulin was decreased but transgelin was increased in ITGA5B1 groups. A recent study reported that the inhibition of thrombospondin- 1 prevents TGF- $\beta 1$ activation and protects against PAH development (29). 
Epiregulin is an epidermal growth factor (EGF)-related growth regulating peptide which involved in vascular SMCs proliferation and induction of SMCs dedifferentiation (30). Inversely, transgelin is ubiquitously expressed in vascular SMCs and is an early marker of SMCs differentiation (31). Our results were consistent with the above reports. The mitigated phenotypic transition in ITGA5B1 groups indicated that rBMSCs/ITGA5B1 maybe the determinants for the regulation SMC phenotype marker expression and involved in the maintenance of the contractile phenotype in HPASMC.

PAH not only characterized as a disease of endothelial dysfunction but also with an imbalance between vasoconstrictors and vasodilators. Thromboxane A2 (TbxA2) and ET-1 are a potent vasoconstrictor while NO has strong vasodilatory effects. TbxA2 was produced by thromboxane synthase (TbxAS) and ultimately stimulates TbxA2 receptor (TbxA2R) to induce vasoconstriction (32). ET-1 is known to be an extremely powerful vasoconstrictor, involved in vascular remodeling and plays a major role in the pathophysiology of hypertension. Supporting the concept that reducing ET-1 vasoconstrictor activity contributes to the improvement in endothelial vasodilator function (33). With the reduction of ET-1, the vasodilator NO production was enhanced in ITGA5B1 groups. In consideration of NO is synthesized largely by eNOS in endothelial cell (27). The present results also support a hypothesis related to the recovery of diastolic-systolic factors balance, such as up-regulated eNOS, NO production and repressed ET-1 protein expression and pro-inflammatory factors.

Taken together, our results reveal a critical role of the rBMSCs/ITGA5B1 on inhibited inflammation, oxidative stress, and HPASMC synthetic phenotype evidenced by lowering HPASMC pro-inflammation factors, alleviating oxidative stress, reversing the synthetic phenotype transition, whereas elevating contractile gene transgelin, restoring the morphological changes of HPASMC. Furthermore, rBMSCs/ITGA5B1 regulated the imbalance between vasoconstrictors and vasodilators induced by MCT. The focus on inflammation, oxidative stress, phenotypic transition, balance between vasoconstrictors and vasodilators in HPASMC, and the way to enhance the anoikis resistance to successful application of stem cell therapy as we mentioned previously (12), may lend itself to a whole new strategy for preventing and potentially reversing $\mathrm{PAH}$ progression in the future.

\section{Acknowledgements}

This work was supported by the Natural Science Foundation of Shandong Province of China (grant number
ZR2016HP33); and the National Natural Science Foundation of China (grant number 81270104). We are grateful to Dr. Li Pan for technical assistance.

\section{Potential Conflict of Interest}

The authors have no conflicting financial interest.

\section{References}

1. Tuder RM. Pulmonary vascular remodeling in pulmonary hypertension. Cell Tissue Res 2017;367:643-649

2. Coll-Bonfill N, de la Cruz-Thea B, Pisano MV, Musri MM. Noncoding RNAs in smooth muscle cell homeostasis: implications in phenotypic switch and vascular disorders. Pflugers Arch 2016;468:1071-1087

3. Chistiakov DA, Orekhov AN, Bobryshev YV. Vascular smooth muscle cell in atherosclerosis. Acta Physiol (Oxf) 2015;214:33-50

4. Crosswhite P, Sun Z. Nitric oxide, oxidative stress and inflammation in pulmonary arterial hypertension. J Hypertens 2010;28:201-212

5. Prudente A, Favaro WJ, Reis LO, Riccetto CL. Nitric oxide coating polypropylene mesh increases angiogenesis and reduces inflammatory response and apoptosis. Int Urol Nephrol 2017;49:597-605

6. Csiszar A, Labinskyy N, Olson S, Pinto JT, Gupte S, Wu JM, Hu F, Ballabh P, Podlutsky A, Losonczy G, de Cabo R, Mathew R, Wolin MS, Ungvari Z. Resveratrol prevents monocrotaline-induced pulmonary hypertension in rats. Hypertension 2009;54:668-675

7. Mónica FZ, Bian K, Murad F. The endothelium-dependent nitric oxide-cGMP pathway. Adv Pharmacol 2016;77:1-27

8. Nickel KF, Laux V, Heumann $R$, von Degenfeld G. Thrombin has biphasic effects on the nitric oxide-cGMP pathway in endothelial cells and contributes to experimental pulmonary hypertension. PLoS One 2013;8: e63504

9. Goto J, Ishikawa K, Kawamura K, Watanabe Y, Matumoto H, Sugawara D, Maruyama Y. Heme oxygenase-1 reduces murine monocrotaline-induced pulmonary inflammatory responses and resultant right ventricular overload. Antioxid Redox Signal 2002;4:563-568

10. Fischer UM, Harting MT, Jimenez F, Monzon-Posadas WO, Xue H, Savitz SI, Laine GA, Cox CS Jr. Pulmonary passage is a major obstacle for intravenous stem cell delivery: the pulmonary first-pass effect. Stem Cells Dev 2009; 18:683-692

11. Nasri F, Mohtasebi MS, Hashemi E, Zarrabi M, Gholijani N, Sarvestani EK. Therapeutic efficacy of mesenchymal stem cells and mesenchymal stem cells-derived neural progenitors in experimental autoimmune encephalomyelitis. Int J Stem Cells 2018;11:68-77

12. Chen HY, Pan L, Yang HL, Xia P, Yu WC, Tang WQ, Zhang YX, Chen SF, Xue YZ, Wang LX. Integrin alpha5betal suppresses rBMSCs anoikis and promotes nitric 
oxide production. Biomed Pharmacother 2018;99:1-8

13. Chen H, Yang H, Yue H, Strappe PM, Xia P, Pan L, Zhang Y, Chai S, Chen S, Ma L, and Wang L. Mesenchymal Stem Cells Expressing eNOS and a Cavl Mutant Inhibit Vascular Smooth Muscle Cell Proliferation in a Rat Model of Pulmonary Hypertension. Heart Lung Circ 2017;26: 509-518

14. Xia P, Chen HY, Chen SF, Wang L, Strappe PM, Yang HL, Zhou CH, Zhang X, Zhang YX, Ma LL, Wang L. The stimulatory effects of eNOS/F92A-Cavl on NO production and angiogenesis in BMSCs. Biomed Pharmacother 2016; 77:7-13

15. Lee S, Choi E, Cha MJ, Hwang KC. Cell adhesion and long-term survival of transplanted mesenchymal stem cells: a prerequisite for cell therapy. Oxid Med Cell Longev 2015; 2015:632902

16. Hood ED, Greineder CF, Dodia C, Han J, Mesaros C, Shuvaev VV, Blair IA, Fisher AB, Muzykantov VR. Antioxidant protection by PECAM-targeted delivery of a novel NADPH-oxidase inhibitor to the endothelium in vitro and in vivo. J Control Release 2012;163:161-169

17. Lincoln TM, Wu X, Sellak H, Dey N, Choi CS. Regulation of vascular smooth muscle cell phenotype by cyclic GMP and cyclic GMP-dependent protein kinase. Front Biosci 2006;11:356-367

18. Ko CY, Chang WC, Wang JM. Biological roles of CCAAT/Enhancer-binding protein delta during inflammation. J Biomed Sci 2015;22:6

19. Feinberg MW, Cao Z, Wara AK, Lebedeva MA, Senbanerjee S, Jain MK. Kruppel-like factor 4 is a mediator of proinflammatory signaling in macrophages. J Biol Chem 2005; 280:38247-38258

20. Autieri MV. Kruppel-like factor 4: transcriptional regulator of proliferation, or inflammation, or differentiation, or all three? Circ Res 2008;102:1455-1457

21. Huang S, Chen P, Shui X, He Y, Wang H, Zheng J, Zhang L, Li J, Xue Y, Chen C, Lei W. Baicalin attenuates transforming growth factor- $\beta$ 1-induced human pulmonary artery smooth muscle cell proliferation and phenotypic switch by inhibiting hypoxia inducible factor- $1 \alpha$ and aryl hydrocarbon receptor expression. J Pharm Pharmacol 2014; 66:1469-1477

22. Iwasaki Y, Suganami T, Hachiya R, Shirakawa I, Kim-Saijo M, Tanaka M, Hamaguchi M, Takai-Igarashi T, Nakai $M$, Miyamoto Y, Ogawa Y. Activating transcription factor 4 links metabolic stress to interleukin-6 expression in macrophages. Diabetes 2014;63:152-161

23. Belhaj A, Dewachter L, Kerbaul F, Brimioulle S, Dewachter C, Naeije R, Rondelet B. Heme oxygenase-1 and inflammation in experimental right ventricular failure on prolonged overcirculation-induced pulmonary hypertension. PLoS One 2013;8:e69470
24. Tamosiuniene R, Manouvakhova O, Mesange P, Saito T, Qian J, Sanyal M, Lin YC, Nguyen LP, Luria A, Tu AB, Sante JM, Rabinovitch M, Fitzgerald DJ, Graham BB, Habtezion A, Voelkel NF, Aurelian L, Nicolls MR. Dominant role for regulatory $\mathrm{T}$ cells in protecting females against pulmonary hypertension. Circ Res 2018;122:16891702

25. Hill MR, Papafili A, Booth H, Lawson P, Hubner M, Beynon H, Read C, Lindahl G, Marshall RP, McAnulty RJ, Laurent GJ. Functional prostaglandin-endoperoxide synthase 2 polymorphism predicts poor outcome in sarcoidosis. Am J Respir Crit Care Med 2006;174:915-922

26. Peroni JF, Borjesson DL. Anti-inflammatory and immunomodulatory activities of stem cells. Vet Clin North Am Equine Pract 2011;27:351-362

27. Lee H, Lee JC, Kwon JH, Kim KC, Cho MS, Yang YS, Oh W, Choi SJ, Seo ES, Lee SJ, Wang TJ, Hong YM. The effect of umbilical cord blood derived mesenchymal stem cells in monocrotaline-induced pulmonary artery hypertension rats. J Korean Med Sci 2015;30:576-585

28. Vaillancourt M, Ruffenach G, Meloche J, Bonnet S. Adaptation and remodelling of the pulmonary circulation in pulmonary hypertension. Can J Cardiol 2015;31:407-415

29. Kumar R, Mickael C, Kassa B, Gebreab L, Robinson JC, Koyanagi DE, Sanders L, Barthel L, Meadows C, Fox D, Irwin D, Li M, McKeon BA, Riddle S, Dale Brown R, Morgan LE, Evans CM, Hernandez-Saavedra D, Bandeira A, Maloney JP, Bull TM, Janssen WJ, Stenmark KR, Tuder RM, Graham BB. TGF- $\beta$ activation by bone marrow-derived thrombospondin-1 causes Schistosoma- and hypoxia-induced pulmonary hypertension. Nat Commun 2017;8: 15494

30. Takahashi M, Hayashi K, Yoshida K, Ohkawa Y, Komurasaki T, Kitabatake A, Ogawa A, Nishida W, Yano M, Monden M, Sobue K. Epiregulin as a major autocrine/paracrine factor released from ERK- and p38MAPKactivated vascular smooth muscle cells. Circulation 2003; 108:2524-2529

31. Salmon M, Gomez D, Greene E, Shankman L, Owens GK. Cooperative binding of KLF4, pELK-1, and HDAC2 to a $\mathrm{G} / \mathrm{C}$ repressor element in the SM22 $\alpha$ promoter mediates transcriptional silencing during SMC phenotypic switching in vivo. Circ Res 2012;111:685-696

32. Chen H. Role of thromboxane A2 signaling in endothelium-dependent contractions of arteries. Prostaglandins Other Lipid Mediat 2018;134:32-37

33. Diehl KJ, Stauffer BL, Dow CA, Bammert TD, Brunjes DL, Greiner JJ, DeSouza CA. Chronic nebivolol treatment suppresses endothelin-1-mediated vasoconstrictor tone in adults with elevated blood pressure. Hypertension 2016;67: 1196-1204 